\title{
Miniaturized Antenna Solution Based on Lossy Planar Microresonators to Conjointly Control Radiation and Selectivity
}

\author{
Biyun Ma, ${ }^{1}$ Anne Chousseaud, ${ }^{2}$ and Serge Toutain ${ }^{2}$ \\ ${ }^{1}$ School of Electronics and Information Engineering, South China University of Technology, 381 Road Wushan, \\ Guangzhou 510640, China \\ ${ }^{2}$ LUNAM University, University of Nantes, IETR CNRS 6164, Polytech Nantes, La Chantrerie, rue Christian Pauc, \\ BP 50609, 44306 Nantes, France
}

Correspondence should be addressed to Biyun Ma; biyun.ma@gmail.com

Received 14 March 2014; Revised 1 September 2014; Accepted 1 September 2014; Published 13 October 2014

Academic Editor: Matteo Pastorino

Copyright (C) 2014 Biyun Ma et al. This is an open access article distributed under the Creative Commons Attribution License, which permits unrestricted use, distribution, and reproduction in any medium, provided the original work is properly cited.

\begin{abstract}
We propose a new method to design miniaturized compact antennas, in which it is possible to control conjointly the radiation efficiency and the bandwidth selectivity of the antenna. And this method has been validated by the realization of prototypes based on planar resonators. The geometry of these resonators has been chosen because their unloaded quality factor can be controlled and is mainly dependent on radiation loss. In the first time, a filter with a significant potential to radiation has been realized by choosing suitable miniaturized resonators. An antenna, based on the same structure, in which the output of the filter was removed (load by air resistance) can be obtained. Modification of the quality factor of each resonator is necessary to take into account the change of the load value from the previous filter to the final structure. The position and the quality factor of the resonators are determined by a filter design concept to obtain a specific frequency response in which each resonator is a basic radiation element. Load of the antenna is ultimately a distributed load constituted by the parallel contributions of each resonators to radiation loss. In other words such an antenna can also be called radiating filter.
\end{abstract}

\section{Introduction}

Due to efforts increasingly important to miniaturize mobile terminals, the volume dedicated to the antenna and the filter function in RF front ends becomes more and more limited. In this context, an important effort on the design of the antenna is needed. Furthermore, in future wireless communication systems, with the proliferation of communication standards with bandwidths increasingly close to each other, higher selectivity is required. So, higher order filtering functions are needed. The specifications of these filtering communication systems are currently met by the use of miniaturized external filters SAW (surface acoustic wave [1]) or BAW (bulk acoustic wave [2]). Considering the overall integration of RF front ends, it seems more and more appropriate to lighten the filtering function itself by distributing it on the other functions of the system, such as LNA (low noise amplifier) or antenna. The simultaneous control of selectivity and radiation performances in a reception or emission chain takes part of solutions to miniaturize the systems. This last point presents also the interest to reduce the interconnections between functions and therefore noise and losses in the system. However, codesign of filtering and radiating structures requires a compromise between bandwidth selectivity and radiation efficiency.

In this paper, we are specifically interested in proposing a solution of compact antenna with the possibility for the antenna to contribute significantly to the filtering performance of the transceiver. Several papers have already been published on antenna/filter codesign [3-10], but studies are limited to the insertion of a filtering function close to, or in, the volume of the antenna, without taking into account the uncontrolled radiation of the filter in the rejected bandwidth. Studies are also limited to the increase of the frequency response selectivity only, without modifying the radiation function. In another example, Bourtoutian et al. [11] propose an improved method to achieve the codesign of a filtering and radiating structure by using the antenna as the last 


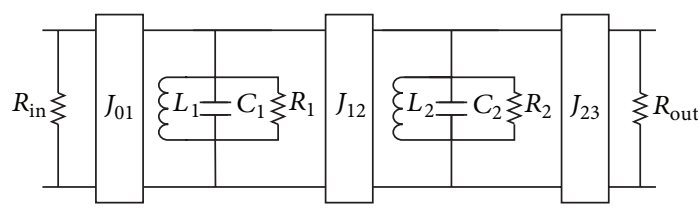

FIgURe 1: Two-pole lossy filter model.

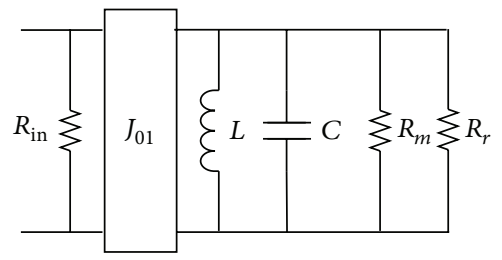

Figure 2: Antenna model.

resonator of the filter. However, the filter resonators have limited radiation influence in this method; the filtering and radiation performances were not optimized simultaneously.

In this context, we are interested in this problem and attempt to define and propose a new design method to improve both of them simultaneously. In order to obtain precise bandwidth selectivity, a filter design concept by predistortion is introduced to take into account resonators' radiation loss in the filtering function. This concept was first proposed by Livingston [12]. We have applied it to a planar structure currently used in SiP (system in package) or SoP (system on package) [13] technologies. We will present the description of the resonator and discuss this method in Sections 2 and 3, and original solution will be presented in Section 4. At last, some conclusions will be derived.

\section{Resonator Characterization}

Resonators are key structures in the design of both filters and antennas [14]. Each one can be completely defined by its resonance frequency and its unload quality factor $Q_{u}(1)$ which depends on the reactive energy stored in the resonator and on loss:

$$
Q_{u}=2 \pi \frac{\text { Energy stored }}{\text { Energy dissipated by cycle }} .
$$

For a resonator in planar technology, dissipated energy is related to the material loss (conductors and dielectric substrate) and the radiation loss. Figure 1 shows the example of a 2nd order lossy filter equivalent circuit, where $R_{n}$ represents the loss in the $n$th resonator $(n=1,2)$. In the same manner, $L_{n}$ and $C_{n}$ are reactive elements linked to resonant frequency. $J_{n m}$ are admittance inverters that represent couplings between $n$th and $m$ th resonators, between input and first resonator, and between last resonator and output. $R_{\text {in }}$ and $R_{\text {out }}$ are input and output impedances.

In the same manner, a planar antenna can be represented by a resonator $L C R_{r} R_{m}$ coupled with an admittance inverter $J_{01}$ modeling the matching circuit of the antenna (Figure 2). In this equivalent circuit, $L$ and $C$ are reactive elements linked

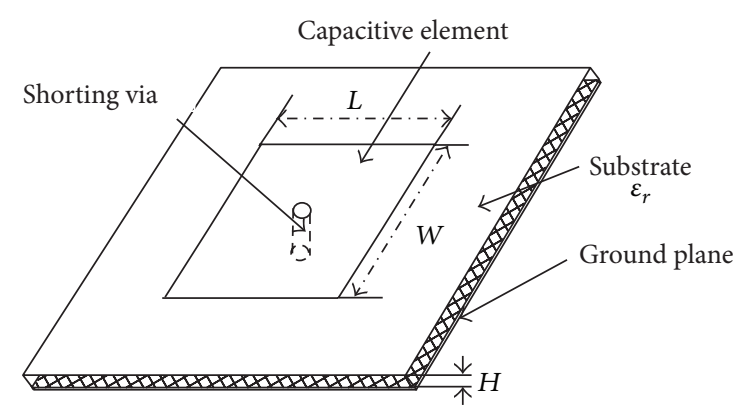

FIgURE 3: Proposed microresonator.

to resonant frequency. $R_{r}$ represents the radiation loss and $R_{m}$ the material loss. $R_{\text {in }}$ is the input impedance.

For the filter design, we focus on the transmission characteristics and try to obtain high unload quality factor resonators by different methods in limited dimensions. For the antenna design, we focus on the matching characteristics and try to obtain high radiation efficiency resonators by different technologies. In other words, the antenna is a kind of filter loaded by air resistance $(377 \Omega$ ), and the resonators are the key structures to control the radiation and the bandwidth selectivity.

We propose to apply the method we propose to the elementary structure of a quasi-lumped planar microresonator constituted by a very short open-ended microstrip line and a shorting via-hole (as shown in Figure 3). Equivalent models for selectivity and radiation behaviors have been proposed previously [15-17].

This microresonator is compact (around $\lambda_{0} / 4 \times \lambda_{0} / 19$ at the resonant frequency) and has a monopole-like radiation pattern. In this kind of structure, the unload quality factor of this resonator is variable by dimensions and is mainly dependent on the radiation loss. It seems quite easy to realize a compromise between radiation behavior and bandwidth selectivity. Furthermore, it is theoretically possible to modify the metallization shape without changing its total area and the via-hole position, without any impact on the resonance frequency. However, the shape of the metallization and the position of the via-hole lead to the possible existence of higher order modes and therefore also to the level of reactive energy stored and to the radiation losses in the resonator.

\section{Solution to Conjointly Control Radiation and Selectivity}

A miniaturized antenna solution is first based on the control of the structure selectivity bandwidth and radiation efficiency, according to the template set for the filtering performances, in which the power supplied to each resonator will be partly radiated. The starting point is then to couple the radiating resonators to obtain the desired bandwidth. Secondly, to control the radiation of each resonator as accurately as possible, radiation losses have to be predominant compared to losses in the materials. This will have an impact on both the materials used and the geometry of the resonator. As 


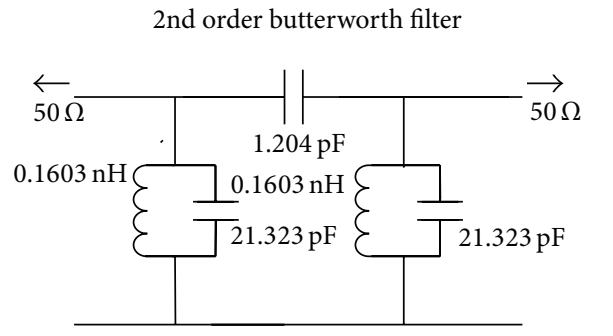

Center frequency $f_{0}=2.65 \mathrm{GHz}, \mathrm{BW}=2.55-2.75 \mathrm{GHz}$

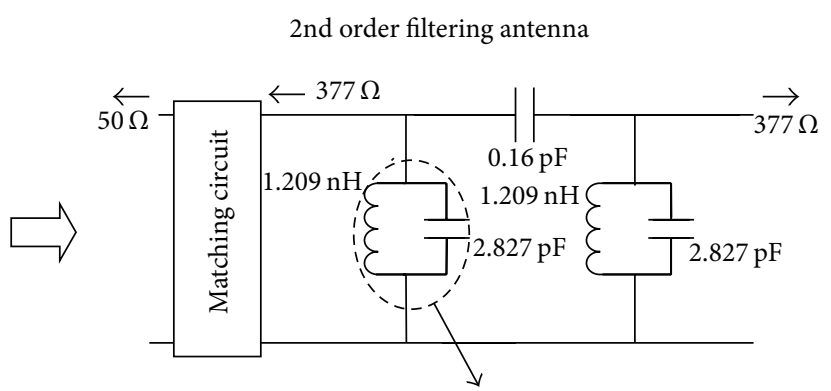

The quasi-lumped microresonators proposed in Section 2 [16] are be used to instead of the LC resonator

FIgURE 4: Schematic diagram that illustrates the solution steps.

the resonators are lossy resonators, predistortion techniques should then be used to control the filtering performance [12]. These techniques are used to compensate the effects of energy dissipation in filters so that their responses can be conformed to ideal filters at the price of higher insertion loss. The loss of each resonator is taken into account in the filter design and the bandwidth is controlled despite these losses.

The proposed design solution is following five steps (see Figure 4).

(1) A suitable geometry of resonator is chosen, in which it is possible to control radiation performances while ensuring miniaturized dimensions. The quality factor of this resonator must be variable and is mainly dependent on the radiation loss. The defined equivalent circuit of this resonator is suitable to use the techniques of classic filter synthesis. Some characteristic of the chosen resonators are necessary to learn about, such as their radiation model and the circuit model with closed-form expressions of the unloaded quality factor. This step is the key point in our solution. For the remainder of the study, we chose the microresonator mentioned in Section 2 as a beginning to validate our solution.

(2) A lossless filter equivalent circuit with selective bandwidth and center frequency clearly defined is designed by a classic filter design method.

(3) The load resistance of the previous filter is changed to $377 \Omega$, which is the air resistance, to model the radiation.

(4) The couplings between each resonator are modified by predistortion techniques to take into account the radiation and material loss while maintaining the bandwidth and selectivity defined in each resonator.

(5) An antenna with conjointly control of radiation and bandwidth selectivity is designed by using the microresonator mentioned in first step.

Considering the coupling of several resonators and placing them in such positions that the energy can be coupled to all resonators within control, it is possible to synthesize a filtering antenna in which each resonator radiates part of the energy until all available energy from the generator is radiated.

In this solution, it is necessary to work concurrently with an electromagnetic approach, to understand and control the various electromagnetic phenomena, and with equivalent model, to define the structure of the basic filter. After the choice of the resonator, the next step is to design a nonradiating filter constituted by several resonators in order to respect the expected bandwidth. In this paper, we propose to design a second-order filter. This step is necessary to validate the equivalent circuit defined for the planar resonator and for the filter.

\section{Experiments and Results}

4.1. Design of a Second-Order Filter Using Controlled Loss Microresonators. To make a filter, it is necessary

(i) to control the resonance frequency and the quality factor of the resonators constituting the filter;

(ii) to control the coupling between filter and generator, between filter and load, and between resonators inside the filter.

A second-order pass-band filter can be designed using two coupled resonators. The coupling is controlled by the gap dimensions (included in $J_{n m}$-inverters in Figure 1) between them and between resonators and I/O feeding lines. For a low loss filter, the resonators are constituted by metallizations above and below a substrate as thin as possible, with the highest possible permittivity to confine electrical field in the dielectric substrate. The shorting via-holes have to be placed at one corner of the metallizations in order to decrease the insertion loss [17]. For several coupled resonators, when two via-holes are positioned on either side of a gap, the optimum position for vias is obtained when vias are located on opposite corners.

From there, the filter is converted into a radiating one. To increase the radiation losses, each elementary resonator must be modified to have the lowest possible quality factor. This requires the use of a dielectric substrate of permittivity as low as possible to avoid confining the electric field in the substrate. This also requires the use of a dielectric substrate with 


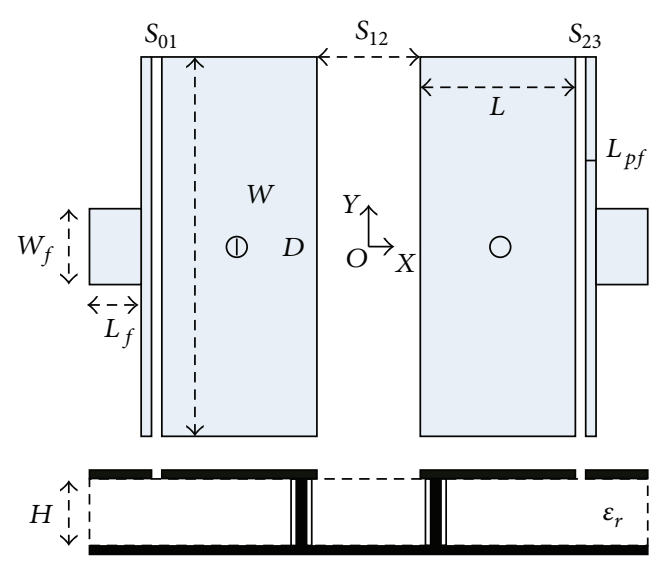

FIGURE 5: Geometry of the radiating filter. (Upper figure is the top view and lower one is side view.) $W_{f}=12 \mathrm{~mm}, L_{f}=5 \mathrm{~mm}, L_{p f}=$ $1 \mathrm{~mm}, S_{01}=S_{23}=2 \mathrm{~mm}$, and $S_{12}=2.9 \mathrm{~mm}$. Dimension: $30 \mathrm{~mm} \times$ $26.3 \mathrm{~mm} \times 3 \mathrm{~mm}\left(\lambda_{0} / 4 \times \lambda_{0} / 5 \times \lambda_{0} / 40\right)$.

the lowest possible losses, such as air or ROHACELL51HF foam (thickness $=3 \mathrm{~mm}, \tan \delta \approx 0$, relative permittivity $=$ 1.06) for example. Another modification is the position of the shorting via-hole on the resonator. We can show that the optimum position of the via-hole to increase radiation is at the center of the resonator because it does not alter the radiation of the slots. The unload $Q$ factor and resonance frequency $f_{0}$ can be obtained theoretically with the radiation model [16]. Predistortion techniques are finally applied to take into account the radiation loss. Figure 5 presents the structure of the proposed radiating filter fabricated on ROHACELL51HF. Its equivalent circuit model is identical to that of Figure 1.

The geometrical and electromagnetic parameters of each resonator are given in Table 1 .

The shape of the measured frequency response (Figure 6) is identical to that of the simulation. The measured center frequency of this filter is $2.6 \mathrm{GHz}$ (difference of about $2 \%$ with the simulated one) with $8 \%$ bandwidth. The insertion loss in the bandwidth is about $2 \mathrm{~dB}$. The bandwidth is slightly different from that of the simulation (approximately $18 \%$ of the relative bandwidth) due to the variation in the level of insertion loss along the bandwidth.

Due to the ability of this filter to radiate and its very small dimensions relative to working wavelengths, it is relatively difficult to completely control the measurement environment. This difficulty, combined with the influence of access connectors [18], may partly explain the ripples on the upper part of the study domain and therefore the variation on the bandwidth.

The radiation performances of this filter, loaded on $50 \Omega$, are shown in Figure 7. The material is lossless and the short circuit is in patch centre, so patch edges radiate like a quasiomnidirectional pattern [16]. The dimensions of each resonator are small enough to be considered as almost localized so the resulting radiation pattern is a quasi-omnidirectional pattern. Finally, resonators are close to each other (around $\left.\lambda_{0} / 40\right)$ so far-field radiation patterns of the resonators are in phase and form the same radiation pattern as a single

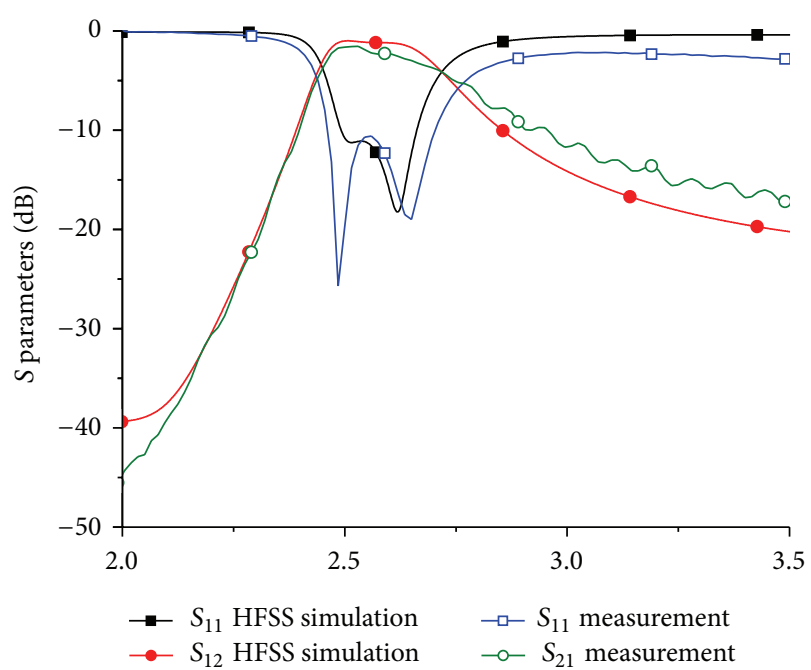

FIGURE 6: $S$ parameters of the proposed radiating filter. Bandwidth in HFSS simulation: [2.53 GHz, $2.70 \mathrm{GHz}$ ], $6.5 \%$. Bandwidth in measurement $(2.50 \mathrm{GHz}, 2.72 \mathrm{GHz}), 8 \%$.

resonator. By this way, the radiation pattern is controllable. The maximum radiation gain is about $3 \mathrm{dBi}$. Figure 7 presents the radiation patterns against the bandwidth. We found that the radiation patterns are similar to each other along the bandwidth with similar gain levels (from \pm 2 to $\pm 5 \mathrm{dBi}$ ). This filter therefore presents strong radiation ability in its passband, and it shows a compromise between radiation pattern and filtering.

With this solution, the energy can be coupled to all of the resonators to realize a filtering function, in which each resonator contributes to the radiation behavior. The insertion loss increase is caused by the radiation loss, since the material loss in this substrate can be neglected.

To finish, in the next section, the structure of the function has to be modified to realize a filtering antenna. The first step is to remove the output. The next step is the modification of the quality factor of each resonator to reflect the variation of the load value between the previous radiating filter and the final antenna.

4.2. Design of a Bandwidth Controllable Antenna by the Modification of Radiating Filter. To switch from the filter to the antenna, it is necessary to remove the output port of the filter. In this case, the structure can still be regarded as a filter for which load is an infinite load resistance. This load is distributed and constituted by the loss of each resonator; the radiating resistance is spread over the different resonators.

So, an antenna is a lossy resonator in which the matching network coupling is represented by an admittance inverter $\left(J_{01}\right.$ in Figure 2) in its equivalent circuit. This matching network presents resistive loss which will reduce the radiated energy.

The proposed approach is to work on the equivalent circuit of the radiating filter proposed in the previous step. A controllable bandwidth antenna can be obtained by modifying the terminal resonator in the equivalent circuit and 
TABLE 1: Geometrical and electromagnetic characteristics of the filter.

Width of the capacitive patch of each resonator

Length of the capacitive patch of each resonator

Diameter of the shorting via-hole

Substrate thickness

Shorting via-hole position

Unload quality factor

Resonance frequency
$W=30 \mathrm{~mm}$

$L=6.2 \mathrm{~mm}$

$D=0.6 \mathrm{~mm}$

$H=3 \mathrm{~mm}$

Center

$Q_{u}=120 \sim 150$ (HFSS simulation) 100 130 (measurement)

$f_{0}=2.76 \mathrm{GHz}$ (HFSS simulation) $2.71 \mathrm{GHz}$ (measurement)
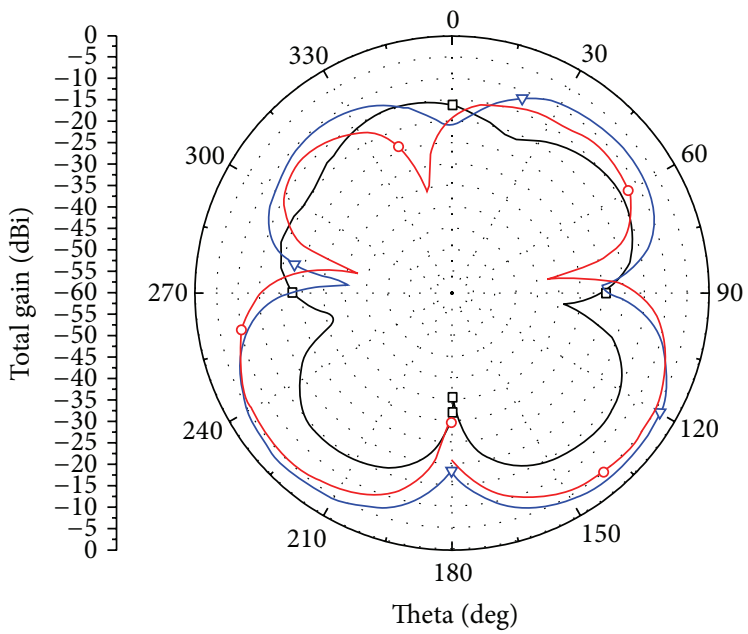

$$
\begin{aligned}
& \rightarrow-2500 \mathrm{MHz} \\
& \rightarrow-2600 \mathrm{MHz} \\
& \multimap-2700 \mathrm{MHz}
\end{aligned}
$$

(a) $\mathrm{Phi}=0^{\circ}$
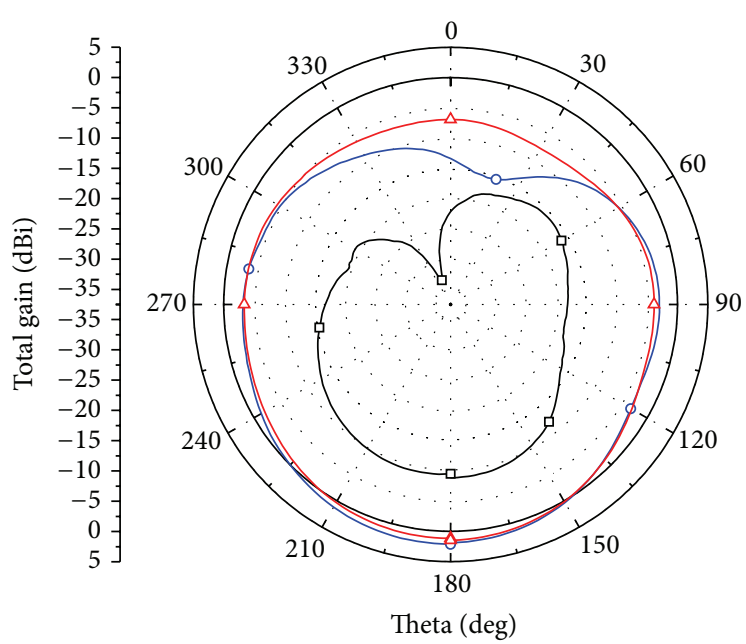

$$
\begin{aligned}
& \multimap-2500 \mathrm{MHz} \\
& \multimap-2600 \mathrm{MHz} \\
& -\triangle 2700 \mathrm{MHz}
\end{aligned}
$$

(b) $\mathrm{Phi}=90^{\circ}$

FIgURE 7: Measured radiation patterns of the radiating filter. 


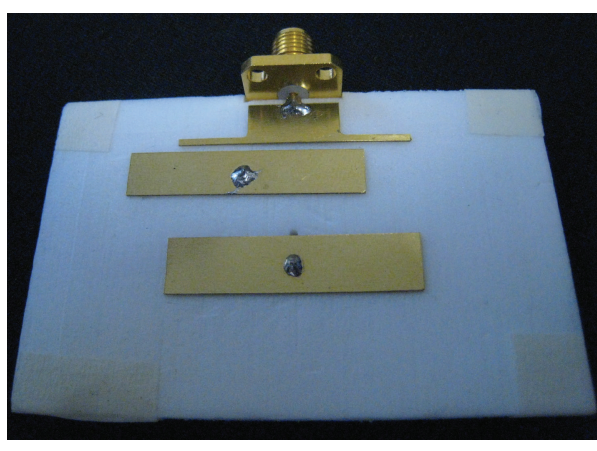

(a) Proposed antenna

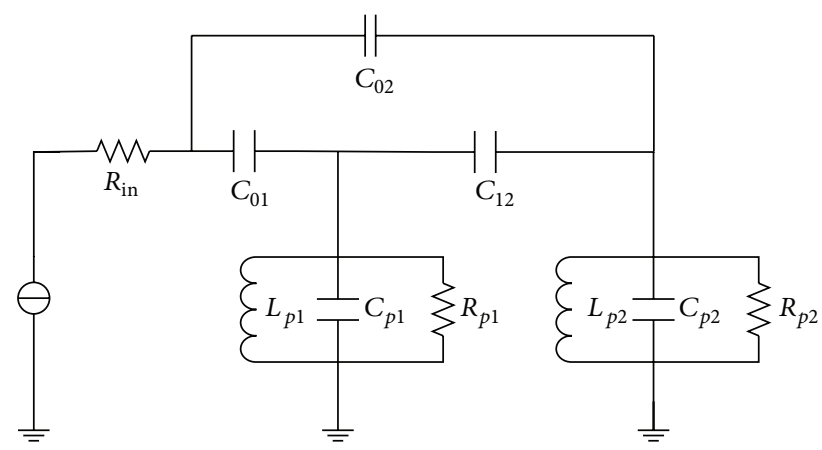

(b) Equivalent circuit of the antenna

FIGURE 8: 2nd order proposed antenna and its equivalent circuit. Dimension: $35 \mathrm{~mm} \times 23 \mathrm{~mm} \times 3 \mathrm{~mm}\left(\lambda_{0} / 3.5 \times \lambda_{0} / 5 \times \lambda_{0} / 40\right)$.

passing on this change on other resonators and on the different couplings. This will change the structure, particularly the position of the resonators relative to each other.

The quality factor of each resonator can be neither too high, which will lead to weak radiation, nor too small, which will lead to weak coupling between resonators and poor bandwidth selectivity.

To increase the radiation efficiency of this structure like for the radiating filter, a thick substrate with low permittivity and low loss has to be chosen, such as ROHACELL51HF or air.

Figure 8 presents the structure of the proposed antenna on ROHACELL51HF and its equivalent circuit model. $R_{p 1}$ and $R_{p 2}$ represent the radiation loss in each resonator. $C_{01}$, $C_{02}$, and $C_{12}$ represent series capacitances induced by the gaps. The dimensions of each resonator are $30 \times 6 \mathrm{~mm}^{2}$ $\left(\lambda_{0} / 4 \times \lambda_{0} / 19\right)$ with center shorting via-holes (diameter $=0.6 \mathrm{~mm}$ ). The measured unload quality factor of these resonators at resonance frequency $2.67 \mathrm{GHz}$ is about $110 \sim 130$. The relative positions of these two resonators are determined by comparing with the frequency response to obtain the desired filter pattern.

Figure 9 presents the reflection coefficient for the proposed structure (HFSS simulation and measurement). The measured antenna presents $6.45 \%$ bandwidth which is similar to the radiating template of the filter. As expected, the antenna center frequencies and bandwidths are very similar (about 1\% discrepancy for the center frequency and $12 \%$ for the relative bandwidth). The presence of a SMA connector was partly taken into account in the simulations.

The far-field radiation pattern of this antenna (Figure 10) is similar to that of the radiating filter and the single resonator [15], with a maximum gain about $4 \mathrm{dBi}$. As shown in Figure 10, the radiation patterns are also similar with frequency in the bandwidth, between $2.55 \mathrm{GHz}$ and $2.75 \mathrm{GHz}$, within $4 \mathrm{dBi}$ difference, but there is an important attenuation in gain level out of the bandwidth, visible at 2.8 and $2.85 \mathrm{GHz}$ especially on Gain Phi $=90^{\circ}$. Figure 11 illustrates the radiation gain versus frequency in different planes cuts. The gain variation versus $\mathrm{Phi}$, in different degrees, proves that the radiation behavior has the same variation trend. Note that there is a transmission zero just before the pass-band, about $2.53 \mathrm{GHz}$ due to the coupling between the input and the second resonator (the same evolution as for the low loss

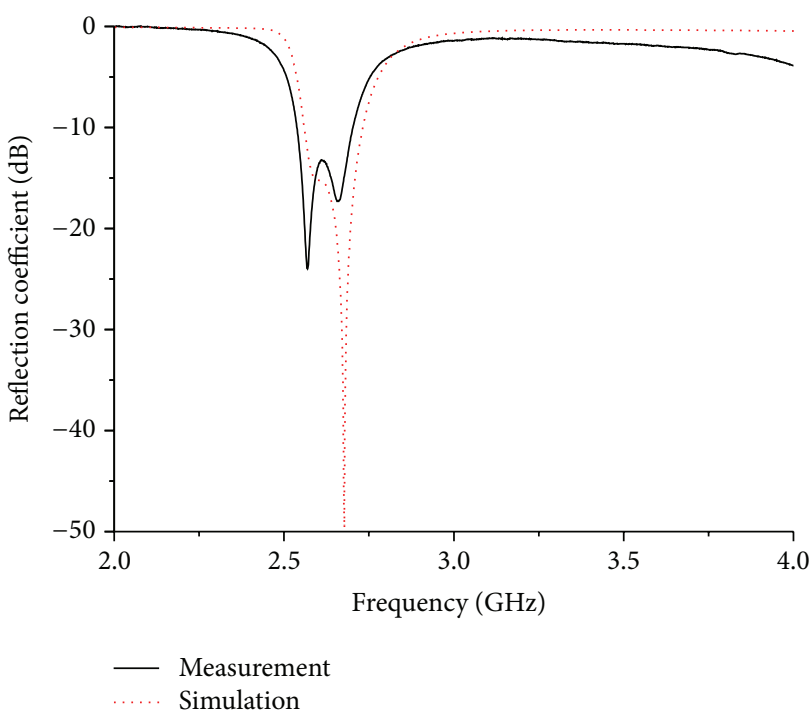

FIGURE 9: Reflection coefficient of the proposed antenna. HFSS simulation bandwidth $(2.58 \mathrm{GHz}, 2.73 \mathrm{GHz}), 5.65 \%$. Measurement bandwidth: [2.55 GHz, $2.72 \mathrm{GHz}], 6.45 \%$.

filter [17]). Each resonator in this antenna is a contributor of filtering and radiation at the same time. This proposed antenna presents filter characteristics; it can be called "filtering antenna" which presents a compromise between radiation efficiency and bandwidth selectivity.

\section{Conclusion}

We have proposed a new miniaturized antenna solution based on planar microresonators. The approach consists in controlling conjointly the radiation efficiency and the bandwidth selectivity of the antenna. Several steps are necessary. First step is the choice of the elementary resonator that must be compact. In this paper, we proposed a planar quasilumped resonator for its geometry relatively simple and its ability to radiate more or less depending on the via-hole position. In a second step, several resonators of this kind are combined to form a nonradiating and then a radiating filter using a predistortion technique. Finally, in order to have 

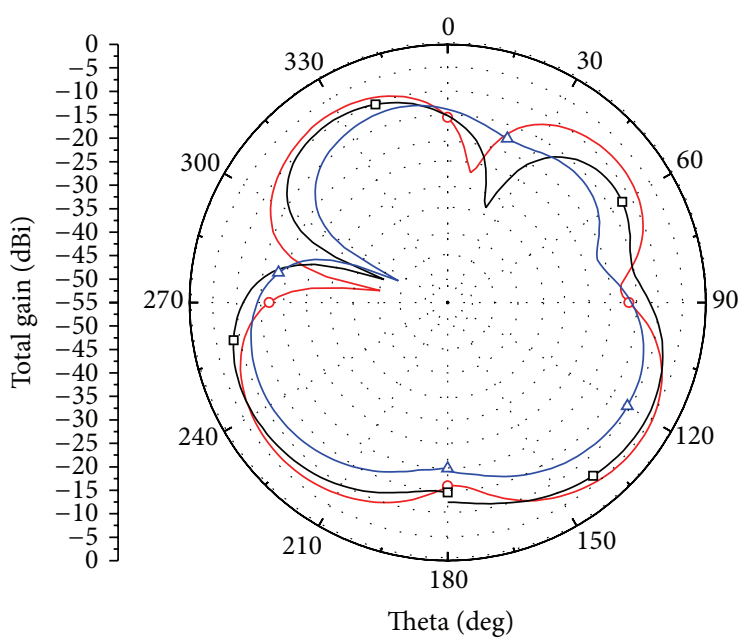

$$
\begin{aligned}
& -02600 \mathrm{MHz} \\
& -\square 2700 \mathrm{MHz} \\
& -\triangle 2800 \mathrm{MHz}
\end{aligned}
$$

(a) $\mathrm{Phi}=0^{\circ}$
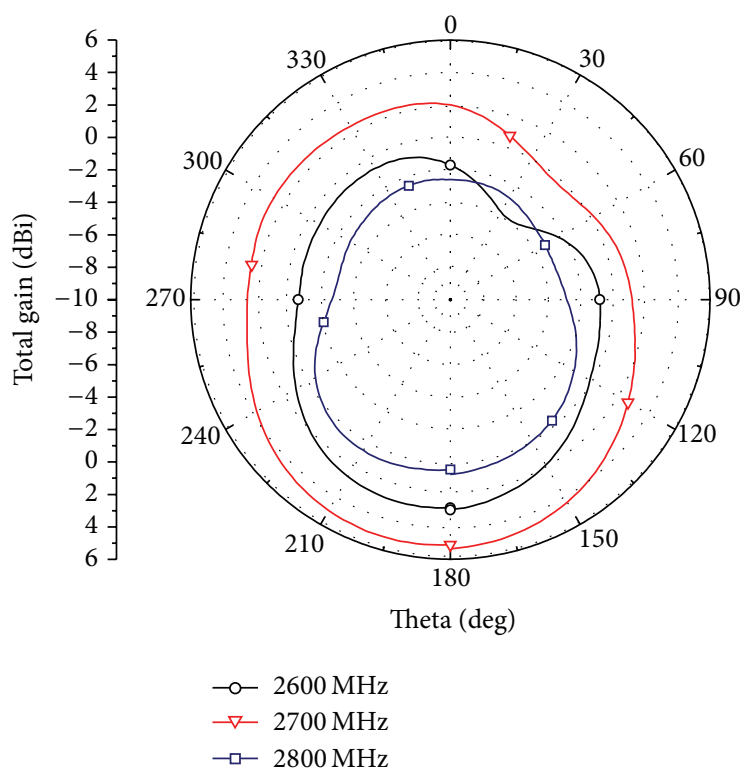

(b) $\mathrm{Phi}=90^{\circ}$

FIGURE 10: Measured radiation patterns of the proposed antenna.

an antenna, such structure has to be modified to become unloaded. In this solution, each resonator is both a radiating element and a matching element for the other resonators to realize the filtering function. A 2nd order planar antenna was successfully realized to validate this approach but the solution can also be used for higher order structures. Thus, synthesized antenna is a compact structure (dimensions of each resonator are $30 \times 6 \mathrm{~mm}^{2}$, i.e., $\lambda_{0} / 4 \times \lambda_{0} / 19$; note that the geometry of the elementary resonator can optimize the dimensions and the filtering function of the target antenna by using other miniaturization technologies, such as LTCC). It presents relatively constant gain and radiation patterns on the frequency bandwidth with a $6.45 \%$ relative bandwidth (quality factor around 110 130) and an important attenuation in gain level out of the bandwidth.

Prospects to this work are twofold. First, the geometry of the elementary resonator can be different from the proposed one, in order to present more couplings between resonators and thus offer improved selectivity for a more compact structure. Furthermore, the ability of this antenna to include a filtering function without increasing the volume of the antenna constitutes also a great advantage for the miniaturization of the communication systems. Indeed, with the proliferation of communication standards with bandwidths increasingly close to each other, higher selectivity is required. Higher order filtering functions are required. It becomes 


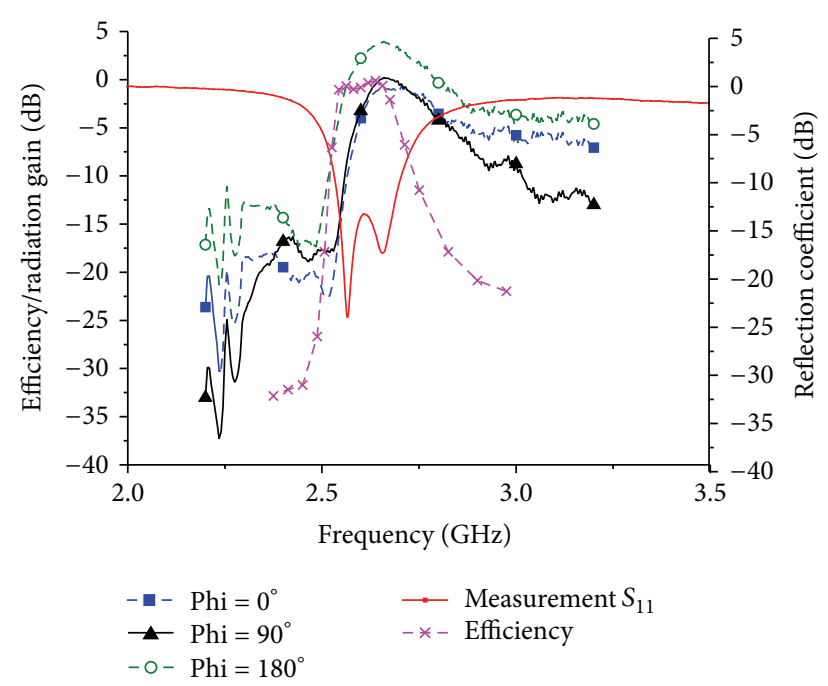

FIGURE 11: Radiation gain versus frequency in different directions cuts.

necessary to lighten the filtering function by distributing it on the other functions of the system. This last point presents also the interest to reduce the interconnections between functions and therefore losses.

\section{Conflict of Interests}

The authors declare that there is no conflict of interests regarding the publication of this paper.

\section{Acknowledgments}

The authors would like to acknowledge the support of French Research National Agency which has financed this work through SRAMM project. Parts of this work were also supported by the National Natural Science Foundation of China (nos. 61401158 and 61302056), National Engineering Technology Research Center for Mobile Ultrasonic Detection (no. 2013FU125X02), the Fundamental Research Funds for the Central Universities of SCUT (nos. 2014ZM0040, 2013ZM0082, and 2014ZB0030), and the Project-sponsored by SRF for ROCS, SEM.

\section{References}

[1] K. Y. Hashimoto, Surface Acoustic Wave Device in Telecommunications: Modeling and Simulation, Springer, New York, NY, USA, 2000.

[2] K. M. Lakin, "Thin film BAW filters for wide bandwidth and high performance applications," in Proceedings of the IEEE MTT-S International Microwave Symposium Digest, vol. 2, pp. 923-926, June 2004.

[3] W. S. Chen and S. C. Wu, "A printed heart monopole antenna with band-rejected characteristics for IEEE 802.16A and UWB applications," Microwave Journal, vol. 50, no. 5, pp. 164-176, 2007.
[4] W.-C. Liu, "Broadband dual-frequency meandered CPW-fed monopole antenna," Electronics Letters, vol. 40, no. 21, pp. 13191320, 2004.

[5] S. Avrillon, Y. Mahé, A. Chousseaud, and S. Toutain, "Multistandard patch antenna based on stepped impedance microstrip," in Proceedings of the IEEE Antennas and Propagation Society International Symposium, vol. 3, pp. 3103-3106, June 2004.

[6] H. K. Lee, J. K. Park, and J. N. Lee, "Design of a planar halfcircle-shaped UWB notch antenna," Microwave and Optical Technology Letters, vol. 47, no. 1, pp. 9-11, 2005.

[7] S. W. Qu, J. L. Li, and Q. Xue, "A band-notched ultrawideband printed monopole antenna," IEEE Antennas and Wireless Propagation Letters, vol. 5, no. 1, pp. 495-498, 2006.

[8] R. Chayono, Y. Kimura, and M. Haneishi, "Design of singlelayer multiband circular MSA with half-ring slots," in Antennas and Propagation Symposium, pp. 2037-2040, 2007.

[9] S. Zheng, Y. Yin, J. Fan, X. Yang, B. Li, and W. Liu, "Analysis of miniature frequency selective surfaces based on fractal antenna-filter-antenna arrays," IEEE Antennas and Wireless Propagation Letters, vol. 11, pp. 240-243, 2012.

[10] A. Abbaspour-Tamijani, K. Sarabandi, and G. M. Rebeiz, "Antenna-filter-antenna arrays as a class of bandpass frequencyselective surfaces," IEEE Transactions on Microwave Theory and Techniques, vol. 52, no. 8, pp. 1781-1789, 2004.

[11] R. Bourtoutian, C. Delaveaud, and S. Toutain, "Novel method for characterizing filtering antennas," IEEE Antennas and Propagation Magazine, vol. 51, no. 3, pp. 209-215, 2009.

[12] R. M. Livingston, "Predistorted waveguide filters for use in communications systems," in Proceedings of the G-MTT International Microwave Symposium, pp. 291-297, IEEE, Dallas, Tex, USA, May 1969.

[13] J. Laskar, S. Pinel, K. Lim et al., "Compact System-on-Package (SOP) architectures for low cost RF front-end modules," in Proceedings of the International Conference on Compound Semiconductor Manufacturing, 2003.

[14] J. S. Hong and M. J. Lancaster, Microstrip Filters for RF/Microwave Applications, Wiley, 2001.

[15] B. Ma, A. Chousseaud, and S. Toutain, "A novel method for filtering antenna design," in Proceedings of the Asia Pacific Microwave Conference (APMC '09), pp. 2660-2663, Singapore, December 2009.

[16] B. Ma, A. Chousseaud, and S. Toutain, "Radiation and circuit model of a planar resonator element with shorting via," IET Microwaves, Antennas \& Propagation, vol. 6, no. 7, pp. 781-787, 2012.

[17] B. Ma, A. Chousseaud, and S. Toutain, "A new design of compact planar microstrip filter," in Proceedings of the International Conference on Electromagnetics in Advanced Applications, (ICEAA '09), pp. 690-693, Torino, Italy, September 2009.

[18] M. Le Pipec, Analyse d'une filière d'interconnexion adaptée aux systèmes de transmissions à haut débit par fibres optiques [Ph.D. thesis], Nantes University, 2007. 

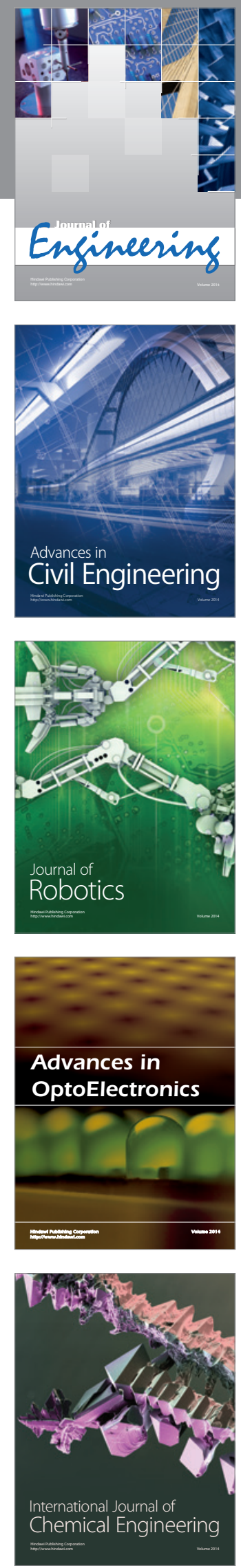

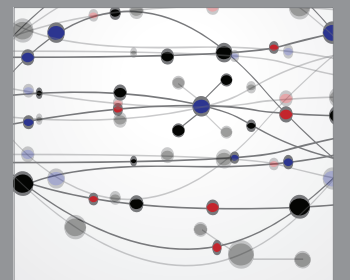

The Scientific World Journal
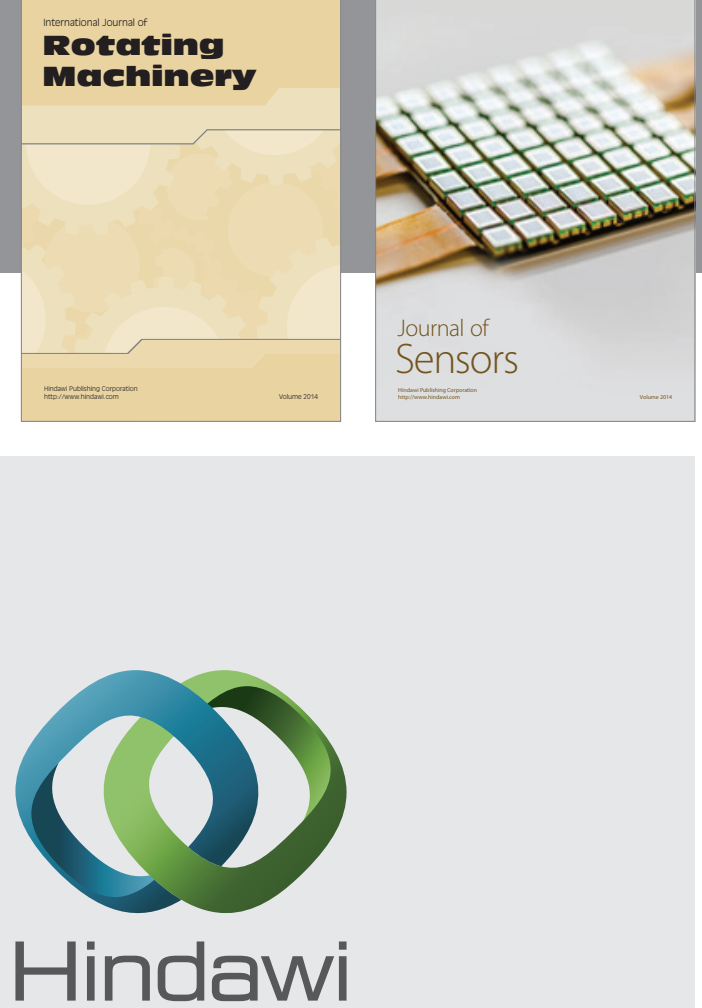

Submit your manuscripts at http://www.hindawi.com
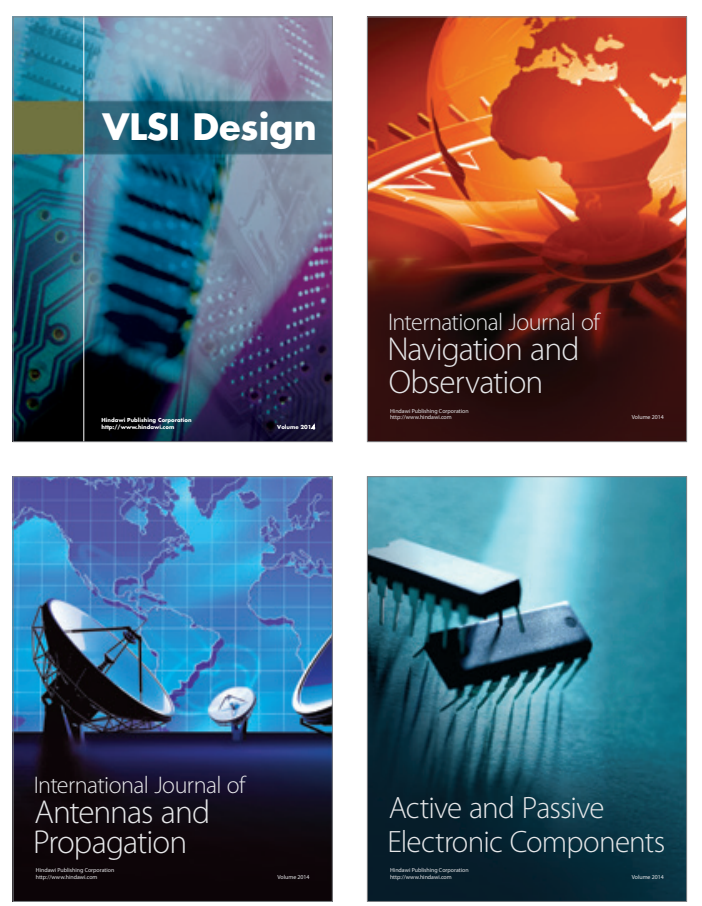
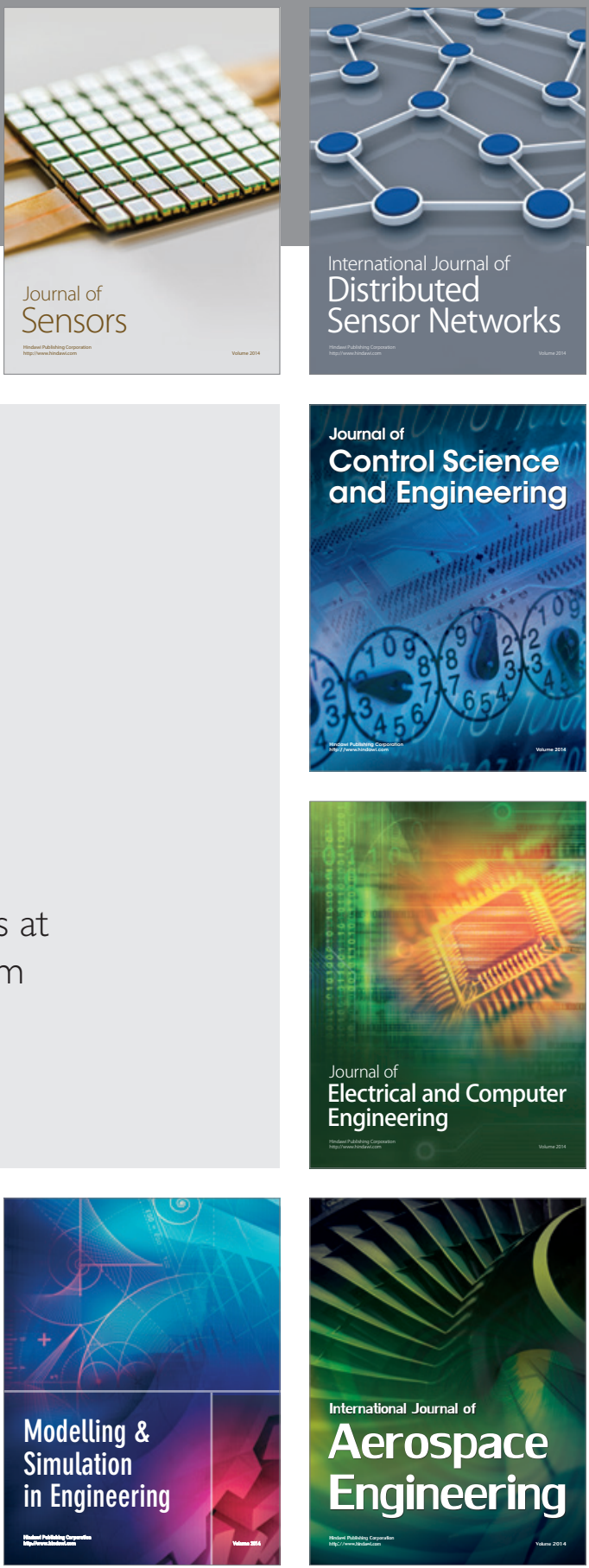

Journal of

Control Science

and Engineering
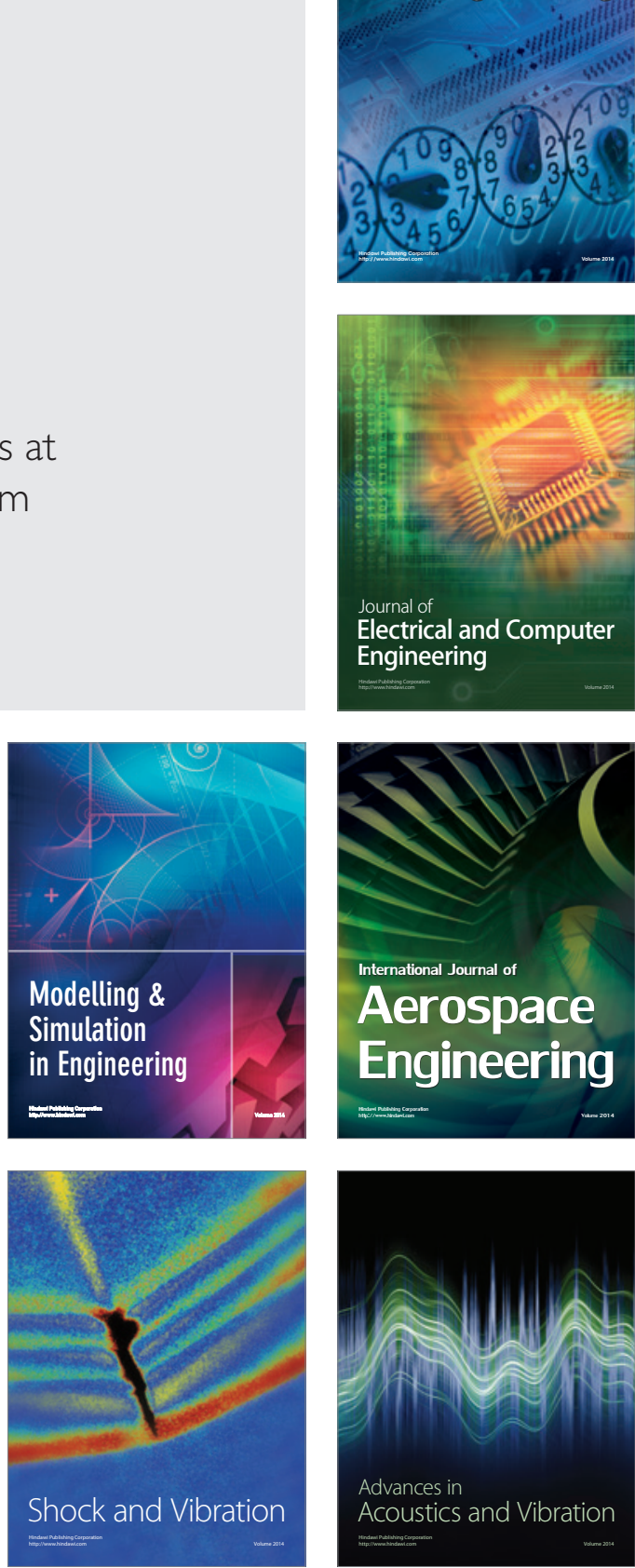